\title{
Implicit Yee-Mesh-Based Finite-Difference Full-Vectorial Beam-Propagation Method
}

\author{
Junji Yamauchi, Member, IEEE, Member, OSA, Takanori Mugita, and Hisamatsu Nakano, Fellow, IEEE
}

\begin{abstract}
A novel Yee-mesh-based finite-difference fullvectorial beam-propagation method is proposed with the aid of an implicit scheme. The efficient algorithm is developed by splitting the propagation axis into two steps. The eigenmode analysis of a rib waveguide is performed using the imaginary-distance procedure. The results show that the present method offers reduction in computational time and memory, while maintaining the same accuracy as the conventional explicit Yee-mesh-based imaginary-distance beam-propagation method. It is demonstrated by the analysis of a polarization converter that the present method can be used for not only the eigenmode analysis but also the propagating beam analysis.
\end{abstract}

Index Terms-Beam-propagation method (BPM), eigenmodes, finite-difference methods, imaginary-distance procedure, implicit schemes, optical waveguides, Yee's mesh.

\section{INTRODUCTION}

$\mathbf{E}$ VALUATION of the modal characteristics of an optical waveguide by numerical analyses is an important issue in the design of photonic integrated circuits. To date, various numerical methods have been proposed for the issue [1]-[4]. One of them is the Yee-mesh-based imaginary-distance beampropagation (BPM) method developed by Lee [4], [5]. This method has the advantage that the obtained eigenmode profiles can be utilized as an incident field of the finite-difference time-domain (FDTD) method, since all the electromagnetic field components located on Yee's mesh are simultaneously analyzed [6].

The Yee-mesh-based imaginary-distance BPM is an explicit scheme owing to the formulation of directly discretizing Maxwell's equations. We refer to this method as EY-BPM. Because of the explicit scheme, the sampling width in the propagation $(z)$ direction must be sufficiently small as compared with those in the transverse ( $x$ and $y$ ) directions so that a stability condition may be satisfied. This leads to decrease in computational efficiency. More unfortunately, the EY-BPM cannot be used in the propagating beam (real-distance) analysis.

On the other hand, the conventional BPM, which is not based on Yee's mesh, is formulated using a Fresnel equation. The Crank-Nicolson (CN) scheme is often applied to the discretization in the $z$ direction of the Fresnel equation. Therefore, the

Manuscript received June 10, 2004; revised February 1, 2005. This work was supported in part by the "University-Industry Joint Research" Project for Private Universities: matching fund subsidy from MEXT, 2003-2007.

J. Yamauchi and H. Nakano are with Faculty of Engineering, Hosei University, Koganei, Tokyo 184-8584, Japan.

T. Mugita is with Fujitsu, Ltd., Tokyo 144-0035, Japan.

Digital Object Identifier 10.1109/JLT.2005.846908
BPM becomes an implicit scheme, leading to unconditional stability. As a result, we can perform efficient computation in both eigenmode and propagating beam analyses. However, for the conventional BPM, the behavior of all the electromagnetic fields cannot be simultaneously analyzed due to the fact that the formulation is based on the wave equation of either an electric or magnetic field.

The use of Yee's mesh is useful for simultaneously evaluating electric and magnetic field components, and the application of the $\mathrm{CN}$ (implicit) scheme is necessary for efficient computation in both eigenmode and propagating beam analyses. When Fresnel-type equations with both electric and magnetic fields are derived, it is expected that the efficient scheme is obtained. However, no attempt has been made for the derivation of the Fresnel-type equations with both electric and magnetic fields.

From this viewpoint, we have recently described the possibility of deriving an implicit Yee-mesh-based finite-difference full-vectorial BPM for the analysis of optical waveguides [7]. In this paper, we present its formulation in detail and show the effectiveness of the present method in both eigenmode and propagating beam analyses.

After deriving finite-difference equations, we perform the eigenmode analysis of a rib waveguide using the imaginary-distance procedure. The efficient algorithm is developed by splitting the propagation axis into two steps. The results reveal that the present method offers reduction in computational time and memory, while maintaining the same accuracy as the conventional EY-BPM. Moreover, the higher order mode is computed using a full-vectorial Gram-Schmidt orthogonalization technique. Finally, it is demonstrated through the analysis of a polarization converter that the present method can also be used for the propagating beam analysis.

\section{FORMULATION}

\section{A. Fresnel-Type Equations}

We consider a linear lossless dielectric medium. The formulation begins with normalized Maxwell's equations in a frequency domain [8]

$$
\begin{aligned}
\nabla \times \tilde{\mathbf{E}} & =-j k_{0} \tilde{\mathbf{H}} \\
\nabla \times \tilde{\mathbf{H}} & =j k_{0} \varepsilon_{r} \tilde{\mathbf{E}} \\
\nabla \cdot \varepsilon_{r} \tilde{\mathbf{E}} & =0 \\
\nabla \cdot \tilde{\mathbf{H}} & =0
\end{aligned}
$$

where $k_{0}$ is the free-space wavenumber and $\varepsilon_{r}$ is the relative permittivity. As a preliminary to the derivation of Fresnel-type 
equations, (1) and (2) are differentiated with respect to $z$. Substituting (3) and (4) into the obtained equations, we get

$$
\begin{aligned}
& \frac{\partial^{2} \tilde{E}_{x}}{\partial z^{2}}+\bar{D}_{x x} \tilde{E}_{x}+\bar{D}_{x y} \tilde{E}_{y}=-j k_{0} \frac{\partial \tilde{H}_{y}}{\partial z} \\
& \frac{\partial^{2} \tilde{E}_{y}}{\partial z^{2}}+\bar{D}_{y y} \tilde{E}_{y}+\bar{D}_{y x} \tilde{E}_{x}=j k_{0} \frac{\partial \tilde{H}_{x}}{\partial z} \\
& \frac{\partial^{2} \tilde{H}_{x}}{\partial z^{2}}+D_{x x} \tilde{H}_{x}+D_{x y} \tilde{H}_{y}=j k_{0} \varepsilon_{r} \frac{\partial \tilde{E}_{y}}{\partial z} \\
& \frac{\partial^{2} \tilde{H}_{y}}{\partial z^{2}}+D_{y y} \tilde{H}_{y}+D_{y x} \tilde{H}_{x}=-j k_{0} \varepsilon_{r} \frac{\partial \tilde{E}_{x}}{\partial z}
\end{aligned}
$$

where

$$
\begin{aligned}
\bar{D}_{a a} \tilde{E}_{a} & =\frac{\partial}{\partial a}\left(\frac{1}{\varepsilon_{r}} \frac{\partial \varepsilon_{r} \tilde{E}_{a}}{\partial a}\right) \\
\bar{D}_{a b} \tilde{E}_{b} & =\frac{\partial}{\partial a}\left(\frac{1}{\varepsilon_{r}} \frac{\partial \varepsilon_{r} \tilde{E}_{b}}{\partial b}\right) \\
D_{a a} \tilde{H}_{a} & =\frac{\partial^{2} \tilde{H}_{a}}{\partial a^{2}} \\
D_{a b} \tilde{H}_{b} & =\frac{\partial^{2} \tilde{H}_{b}}{\partial a \partial b}
\end{aligned}
$$

with $a, b \in\{x, y\}$. Note that the change of the waveguide structure in the $z$ direction is assumed to be small, i.e., $\partial \varepsilon_{r} / \partial z \cong 0$ (this assumption is completely satisfied for the eigenmode analysis with the imaginary-distance procedure).

We now express the transverse ( $x$ or $y$ ) electromagnetic field component $\tilde{\phi}(x, y, z)$ as

$$
\tilde{\phi}(x, y, z)=\phi(x, y, z) \exp \left(-j \beta_{\mathrm{ref}} z\right)
$$

where $\phi(x, y, z)$ is the field component with the slowly varying envelope function of $z, \beta_{\text {ref }}\left(=k_{0} n_{0}\right)$ is the reference propagation constant representing the fast-varying spatial phase, and $n_{0}$ is the reference index. After applying (9) to (5)-(8) and eliminating the second derivatives with respect to $z$ by the slowly varying envelope approximation

$$
\left|\frac{\partial^{2} \phi}{\partial z^{2}}\right| \ll 2 \beta_{\text {ref }}\left|\frac{\partial \phi}{\partial z}\right|
$$

we obtain the following Fresnel-type equations

$$
\begin{aligned}
L_{1} E_{x}+\bar{D}_{x x} E_{x}+\bar{D}_{x y} E_{y} & =-L_{2} H_{y} \\
L_{1} E_{y}+\bar{D}_{y y} E_{y}+\bar{D}_{y x} E_{x} & =L_{2} H_{x} \\
L_{1} H_{x}+D_{x x} H_{x}+D_{x y} H_{y} & =\varepsilon_{r} L_{2} E_{y} \\
L_{1} H_{y}+D_{y y} H_{y}+D_{y x} H_{x} & =-\varepsilon_{r} L_{2} E_{x}
\end{aligned}
$$

where

$$
\begin{aligned}
& L_{1} \phi=-2 j \beta_{\mathrm{ref}} \frac{\partial \phi}{\partial z}-\beta_{\mathrm{ref}}^{2} \phi \\
& L_{2} \phi=j k_{0}\left(\frac{\partial \phi}{\partial z}-j \beta_{\mathrm{ref}} \phi\right) .
\end{aligned}
$$

\section{B. Finite-Difference Equations}

We next derive the finite-difference equations of the Fresneltype equations. Splitting the propagation axis into two steps, (10)-(13) can be written as

[first step] $(z \Rightarrow z+\Delta z / 2)$

$$
\begin{aligned}
L_{1} E_{x}^{l+\frac{1}{4}}+\bar{D}_{x x} E_{x}^{l+\frac{1}{2}}+\bar{D}_{x y} E_{y}^{l} & =-L_{2} H_{y}^{l+\frac{1}{4}} \\
L_{1} E_{y}^{l+\frac{1}{4}}+\bar{D}_{y y} E_{y}^{l}+\bar{D}_{y x} E_{x}^{l+\frac{1}{2}} & =L_{2} H_{x}^{l+\frac{1}{4}} \\
L_{1} H_{x}^{l+\frac{1}{4}}+D_{x x} H_{x}^{l+\frac{1}{2}}+D_{x y} H_{y}^{l+\frac{1}{2}} & =\varepsilon_{r} L_{2} E_{y}^{l+\frac{1}{4}} \\
L_{1} H_{y}^{l+\frac{1}{4}}+D_{y y} H_{y}^{l}+D_{y x} H_{x}^{l} & =-\varepsilon_{r} L_{2} E_{x}^{l+\frac{1}{4}}
\end{aligned}
$$

[second step] $(z+\Delta z / 2 \Rightarrow z+\Delta z)$

$$
\begin{aligned}
L_{1} E_{x}^{l+\frac{3}{4}}+\bar{D}_{x x} E_{x}^{l+\frac{1}{2}}+\bar{D}_{x y} E_{y}^{l+1} & =-L_{2} H_{y}^{l+\frac{3}{4}} \\
L_{1} E_{y}^{l+\frac{3}{4}}+\bar{D}_{y y} E_{y}^{l+1}+\bar{D}_{y x} E_{x}^{l+\frac{1}{2}} & =L_{2} H_{x}^{l+\frac{3}{4}} \\
L_{1} H_{x}^{l+\frac{3}{4}}+D_{x x} H_{x}^{l+\frac{1}{2}}+D_{x y} H_{y}^{l+\frac{1}{2}} & =\varepsilon_{r} L_{2} E_{y}^{l+\frac{3}{4}} \\
L_{1} H_{y}^{l+\frac{3}{4}}+D_{y y} H_{y}^{l+1}+D_{y x} H_{x}^{l+1} & =-\varepsilon_{r} L_{2} E_{x}^{l+\frac{3}{4}}
\end{aligned}
$$

where $z=l \Delta z$ and $\Delta z$ is the sampling width in the $z$ direction. Note that $\partial \phi / \partial z$ in $L_{1 \text { (or 2) }} \phi^{l+(1 / 4)(\text { or (3/4)) means }}$ $\partial \phi /\left.\partial z\right|_{l+(1 / 4)(\text { or }(3 / 4))}$. The $\mathrm{CN}$ scheme is applied to (14)-(21), and the derivatives in the transverse directions are discretized by the central finite difference. As a result, the first-step equations become

$$
\begin{aligned}
& \text { [first step] }(l \Rightarrow l+1 / 2) \\
& \left(\bar{\delta}_{x}^{2}-B_{+}\right) E_{x i+\frac{1}{2}, j}^{l+\frac{1}{2}}+B_{-} E_{x i+\frac{1}{2}, j}^{l}+\bar{\delta}_{x y} E_{y i+\frac{1}{2}, j}^{l} \\
& =-A_{-} H_{y i+\frac{1}{2}, j}^{l+\frac{1}{2}}+A_{+} H_{y i+\frac{1}{2}, j}^{l} \\
& -B_{+} E_{y i, j+\frac{1}{2}}^{l+\frac{1}{2}}+B_{-} E_{y i, j+\frac{1}{2}}^{l}+\bar{\delta}_{y}^{2} E_{y i, j+\frac{1}{2}}^{l}+\bar{\delta}_{y x} E_{x i, j+\frac{1}{2}}^{l+\frac{1}{2}} \\
& =A_{-} H_{x i, j+\frac{1}{2}}^{l+\frac{1}{2}}-A_{+} H_{x i, j+\frac{1}{2}}^{l} \\
& \left(\delta_{x}^{2}-B_{+}\right) H_{x i, j+\frac{1}{2}}^{l+\frac{1}{2}}+B_{-} H_{x i, j+\frac{1}{2}}^{l}+\delta_{x y} H_{y i, j+\frac{1}{2}}^{l+\frac{1}{2}} \\
& =A_{-} \varepsilon_{r i, j+\frac{1}{2}} E_{y i, j+\frac{1}{2}}^{l+\frac{1}{2}}-A_{+} \varepsilon_{r i, j+\frac{1}{2}} E_{y i, j+\frac{1}{2}}^{l} \\
& -B_{+} H_{y i+\frac{1}{2}, j}^{l+\frac{1}{2}}+B_{-} H_{y i+\frac{1}{2}, j}^{l}+\delta_{y}^{2} H_{y i+\frac{1}{2}, j}^{l}+\delta_{y x} H_{x i+\frac{1}{2}, j}^{l} \\
& =-A_{-} \varepsilon_{r i+\frac{1}{2}, j} E_{x i+\frac{1}{2}, j}^{l+\frac{1}{2}}+A_{+} \varepsilon_{r i+\frac{1}{2}, j} E_{x i+\frac{1}{2}, j}^{l}
\end{aligned}
$$

where

$$
\begin{aligned}
& A_{ \pm}=j k_{0}\left(2 / \Delta z \pm j \beta_{\text {ref }} / 2\right) \\
& B_{ \pm}=4 j \beta_{\text {ref }} / \Delta z \pm \beta_{\text {ref }}^{2} / 2
\end{aligned}
$$

and $\delta_{a}^{2}, \bar{\delta}_{a}^{2}, \delta_{a b}, \bar{\delta}_{a b}(a, b \in\{x, y\})$ are the difference operators (see Appendix). Interestingly, the finite-difference equation of $\bar{\delta}_{x}^{2}$ or $\bar{\delta}_{y}^{2}$ based on Yee's mesh coincides with Stern's formula [9]. The discretized forms of the mixed derivatives appear to be equivalent to those derived by Huang and $\mathrm{Xu}$ [10]. However, since Yee's mesh is employed, they differ from those in [10] in that the relative permittivity at an interface and a corner is naturally given by the average of the neighbor ones [11]. 
It should be noted that (22) and (25) form simultaneous equations with unknowns of $E_{x i+(1 / 2), j}^{l+(1 / 2)}$ and $H_{y i+(1 / 2), j}^{l+(1 / 2)}$. They can, therefore, be solved by a substitution technique. Once $E_{x i+(1 / 2), j}^{l+(1 / 2)}$ and $H_{y i+(1 / 2), j}^{l+(1 / 2)}$ are determined, they are substituted into (23) and (24), resulting in simultaneous equations with unknowns of $E_{y i, j+(1 / 2)}^{l+(1 / 2)}$ and $H_{x i, j+(1 / 2)}^{l+(1 / 2)}$, which can again be solved. As a result, we obtain

[first step] $(l \Rightarrow l+1 / 2)$

$$
\begin{aligned}
\left(A_{-}^{2} \varepsilon_{r i+\frac{1}{2}, j}-B_{+}^{2}+B_{+} \bar{\delta}_{x}^{2}\right) E_{x i+\frac{1}{2}, j}^{l+\frac{1}{2}} & \\
= & \left(A_{+} A_{-} \varepsilon_{r i+\frac{1}{2}, j}-B_{+} B_{-}\right) E_{x i+\frac{1}{2}, j}^{l} \\
& \quad-\left(A_{-} B_{-}-A_{+} B_{+}+A_{-} \delta_{y}^{2}\right) H_{y i+\frac{1}{2}, j}^{l} \\
& \quad-B_{+} \bar{\delta}_{x y} E_{y i+\frac{1}{2}, j}^{l}-A_{-} \delta_{y x} H_{x i+\frac{1}{2}, j}^{l} \\
E_{y i, j+\frac{1}{2}}^{l+\frac{1}{2}} & \\
= & \frac{1}{B_{+}}\left\{\left(B_{-}+\bar{\delta}_{y}^{2}\right) E_{y i, j+\frac{1}{2}}^{l}-A_{-} H_{x i, j+\frac{1}{2}}^{l+\frac{1}{2}}\right. \\
& \left.+A_{+} H_{x i, j+\frac{1}{2}}^{l}+\bar{\delta}_{y x} E_{x i, j+\frac{1}{2}}^{l+\frac{1}{2}}\right\} \\
\left(A_{-}^{2} \varepsilon_{r i, j+\frac{1}{2}}-B_{+}^{2}+B_{+} \delta_{x}^{2}\right) H_{x i, j+\frac{1}{2}}^{l+\frac{1}{2}} & \left(A_{+} A_{-} \varepsilon_{r i, j+\frac{1}{2}}-B_{+} B_{-}\right) H_{x i, j+\frac{1}{2}}^{l} \\
\quad+ & \left(A_{-} B_{-}-A_{+} B_{+}+A_{-} \bar{\delta}_{y}^{2}\right) \varepsilon_{r i, j+\frac{1}{2}} E_{y i, j+\frac{1}{2}}^{l} \\
& \quad-B_{+} \delta_{x y} H_{y i, j+\frac{1}{2}}^{l+\frac{1}{2}}+A_{-} \varepsilon_{r i, j+\frac{1}{2}} \bar{\delta}_{y x} E_{x i, j+\frac{1}{2}}^{l+\frac{1}{2}} \\
H_{y i+\frac{1}{2}, j}^{l+\frac{1}{2}} & \frac{1}{B_{+}}\left\{\left(B_{-}+\delta_{y}^{2}\right) H_{y i+\frac{1}{2}, j}^{l}+A_{-} \varepsilon_{r i+\frac{1}{2}, j} E_{x i+\frac{1}{2}, j}^{l+\frac{1}{2}}\right. \\
\quad & \left.-A_{+} \varepsilon_{r i+\frac{1}{2}, j} E_{x i+\frac{1}{2}, j}^{l}+\delta_{y x} H_{x i+\frac{1}{2}, j}^{l}\right\} .
\end{aligned}
$$

Similar procedures are also made for the second step so that we finally obtain

[second step] $(l+1 / 2 \Rightarrow l+1)$

$$
\begin{aligned}
E_{x i+\frac{1}{2}, j}^{l+1} & \\
= & \frac{1}{B_{+}}\left\{\left(B_{-}+\bar{\delta}_{x}^{2}\right) E_{x i+\frac{1}{2}, j}^{l+\frac{1}{2}}+A_{-} H_{y i+\frac{1}{2}, j}^{l+1}\right. \\
& \left.\quad-A_{+} H_{y i+\frac{1}{2}, j}^{l+\frac{1}{2}}+\bar{\delta}_{x y} E_{y i+\frac{1}{2}, j}^{l+1}\right\} \\
\left(A_{-}^{2} \varepsilon_{r i, j+\frac{1}{2}}-B_{+}^{2}+B_{+} \bar{\delta}_{y}^{2}\right) E_{y i, j+\frac{1}{2}}^{l+1} & \\
= & \left(A_{+} A_{-} \varepsilon_{r i, j+\frac{1}{2}}-B_{+} B_{-}\right) E_{y i, j+\frac{1}{2}}^{l+\frac{1}{2}} \\
& +\left(A_{-} B_{-}-A_{+} B_{+}+A_{-} \delta_{x}^{2}\right) H_{x i, j+\frac{1}{2}}^{l+\frac{1}{2}} \\
& -B_{+} \bar{\delta}_{y x} E_{x i, j+\frac{1}{2}}^{l+\frac{1}{2}}+A_{-} \delta_{x y} H_{y i, j+\frac{1}{2}}^{l+\frac{1}{2}}
\end{aligned}
$$

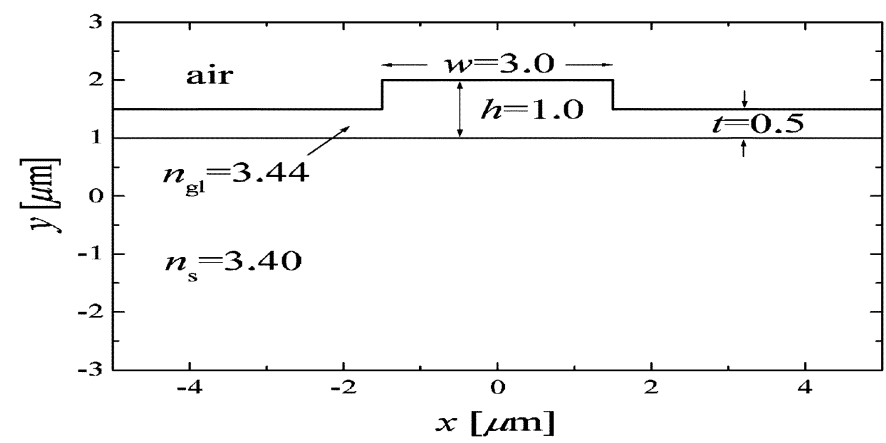

Fig. 1. Configuration of a rib waveguide.

$$
\begin{aligned}
& H_{x i, j+\frac{1}{2}}^{l+1} \\
& =\frac{1}{B_{+}}\left\{\left(B_{-}+\delta_{x}^{2}\right) H_{x i, j+\frac{1}{2}}^{l+\frac{1}{2}}-A_{-} \varepsilon_{r i, j+\frac{1}{2}} E_{y i, j+\frac{1}{2}}^{l+1}\right. \\
& \left.+A_{+} \varepsilon_{r i, j+\frac{1}{2}} E_{y i, j+\frac{1}{2}}^{l+\frac{1}{2}}+\delta_{x y} H_{y i, j+\frac{1}{2}}^{l+\frac{1}{2}}\right\} \\
& \left(A_{-}^{2} \varepsilon_{r i+\frac{1}{2}, j}-B_{+}^{2}+B_{+} \delta_{y}^{2}\right) H_{y i+\frac{1}{2}, j}^{l+1} \\
& =\left(A_{+} A_{-} \varepsilon_{r i+\frac{1}{2}, j}-B_{+} B_{-}\right) H_{y i+\frac{1}{2}, j}^{l+\frac{1}{2}} \\
& \text { - }\left(A_{-} B_{-}-A_{+} B_{+}+A_{-} \bar{\delta}_{x}^{2}\right) \varepsilon_{r i+\frac{1}{2}, j} E_{x i+\frac{1}{2}, j}^{l+\frac{1}{2}} \\
& \text { - } B_{+} \delta_{y x} H_{x i+\frac{1}{2}, j}^{l+1}-A_{-} \varepsilon_{r i+\frac{1}{2}, j} \bar{\delta}_{x y} E_{y i+\frac{1}{2}, j}^{l+1}
\end{aligned}
$$

In the present method, the above two-step equations are solved, updating the solution in the $z$ direction. Efficient computation can be carried out by the Thomas algorithm [14] since (26), (28), (31), and (33) are tridiagonal systems of equations. The others are explicitly solved. Eventually, the calculation is performed with the following process:

$(26) \rightarrow(29) \rightarrow(28) \rightarrow(27) \rightarrow(31) \rightarrow(32) \rightarrow(33) \rightarrow(30)$.

\section{EIGENMODE ANALYSIS}

\section{A. Confirmation of Validity}

In order to confirm the validity of the present method, we perform the eigenmode analysis using the imaginary-distance procedure [2], [15], [16]. The imaginary-distance procedure is a method to extract modal profiles by replacing the real axis $(z)$ with the imaginary axis $(j \tau)$. In the procedure, the propagation constant can be calculated by (34), shown at the bottom of the page, in which the weighting function $|\phi(x, y, \tau)|^{2}$ is adopted to minimize numerical error [4]. Moreover, a technique for successively updating $\beta_{\text {ref }}$ at each propagation step is used [11].

We choose the rib waveguide illustrated in Fig. 1, which is well known as a classical benchmark [3]. The configuration parameters are $n_{\mathrm{s}}=3.40, n_{\mathrm{gl}}=3.44, w=3.0 \mu \mathrm{m}, h=1.0 \mu \mathrm{m}$, and $t=0.5 \mu \mathrm{m}$. The wavelength is taken to be $\lambda=1.15 \mu \mathrm{m}$. The sampling width in the propagation direction is chosen to

$$
\beta(\tau)=\beta_{\text {ref }}+\frac{\iint\{\ln \phi(x, y, \tau+\Delta \tau)-\ln \phi(x, y, \tau)\}|\phi(x, y, \tau)|^{2} d x d y}{\Delta \tau \iint|\phi(x, y, \tau)|^{2} d x d y}
$$




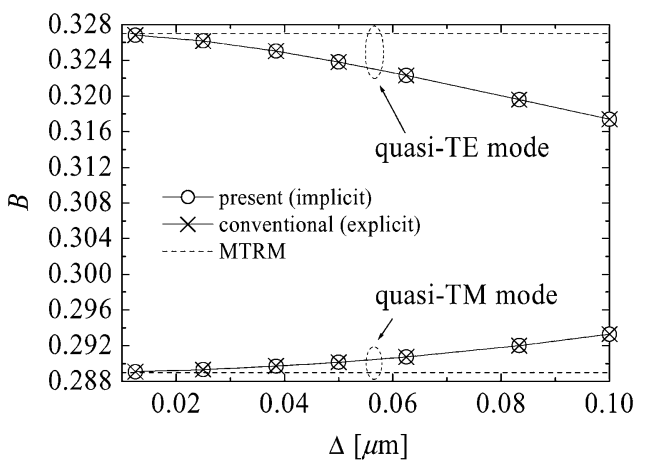

Fig. 2. Normalized propagation constant $B$ versus transverse sampling width.

be $\Delta \tau=1.0 \mu \mathrm{m}$. The transverse sampling width is defined as $\Delta(=\Delta x=\Delta y)$. The computational domain is fixed to be $L_{x} \times L_{y}=10.0 \mu \mathrm{m} \times 6.0 \mu \mathrm{m}$.

For the eigenmode analysis based on the imaginary-distance procedure, we can use various boundary conditions at the edge of the computational region. Needless to say, absorbing boundary conditions, such as the transparent boundary condition [12] and the perfectly matched layers (PMLs) [13], are effective for efficient computation. Nevertheless, in this section, we intentionally use the Dirichlet condition $\phi=0$ to fairly compare several methods, since the computational time and memory are often affected by the boundary conditions to be used. Note that the PML will be used in Section IV.

As a measure to evaluate the validity of the present method, we utilize the normalized propagation constant $B$ defined as $B=\left(n_{\text {eff }}^{2}-n_{\mathrm{s}}^{2}\right) /\left(n_{\mathrm{gl}}^{2}-n_{\mathrm{s}}^{2}\right)$, where $n_{\mathrm{eff}}\left(=\beta / k_{0}\right)$ is the effective index.

Fig. 2 shows $B$ as a function of $\Delta$ for quasi-transverse electric (TE) and quasi-transverse magnetic (TM) modes. For comparison, the results obtained from the conventional EY-BPM and the modal transverse resonance method (MTRM) [3] are also shown in Fig. 2 (note that the four-digit values of the MTRM are believed to be exact [3]). From the figure, we can see that the result of the present method agrees well with that of the conventional EY-BPM. Furthermore, it is found that $B$ obtained by the present method approaches that given by the MTRM as $\Delta$ is decreased. For the conventional EY-BPM, $\Delta \tau$ must be selected in such a way that the stability condition is satisfied. The stability condition is $\Delta \tau<\Delta / \sqrt{2}$ in the three-dimensional analysis [4]. In this paper, $\Delta \tau$ is set to be the upper limit of the stability condition.

The effects of $\Delta \tau$ on the numerical convergence are presented in Fig. 3, in which $\Delta$ is fixed to be $0.05 \mu \mathrm{m}$. It is found that the convergence rate becomes faster as $\Delta \tau$ is increased. For the explicit scheme, however, we cannot choose a larger $\Delta \tau$ due to the exsistence of the stability condition $(\Delta \tau=0.035 \mu \mathrm{m}$ is the upper limit). It is worth mentioning that the implicit scheme allows us to employ a larger $\Delta \tau$ with subsequent faster convergence.

\section{B. Effectiveness of Splitting the Propagation Axis}

In the present method, the propagation axis is split into two steps in order to obtain the tridiagonal systems. In this section, we also present finite-difference equations without splitting and investigate the effect of splitting the propagation axis on accuracy and efficiency in order to validate the splitting procedure.
Here, each method is called a split-type or unsplit-type method, depending on whether the propagation axis is split or not.

We summarize the unsplit-type method. Directly discretizing (10)-(13) by the central finite difference with the CN scheme, we obtain

$$
\begin{aligned}
& \left(b_{+}^{2}-a_{-}^{2} \varepsilon_{r i+\frac{1}{2}, j}-\frac{b_{+}}{2} \bar{\delta}_{x}^{2}-\frac{b_{+}}{2} \delta_{y}^{2}+\frac{1}{4} \delta_{y}^{2} \bar{\delta}_{x}^{2}\right) E_{x i+\frac{1}{2}, j}^{l+1} \\
& =\left(b_{+} b_{-}-a_{+} a_{-} \varepsilon_{r i+\frac{1}{2}, j}\right. \\
& \left.+\frac{b_{+}}{2} \bar{\delta}_{x}^{2}-\frac{b_{-}}{2} \delta_{y}^{2}-\frac{1}{4} \delta_{y}^{2} \bar{\delta}_{x}^{2}\right) E_{x i+\frac{1}{2}, j}^{l} \\
& -\left(a_{+} b_{+}-a_{-} b_{-}-\frac{a_{+}+a_{-}}{2} \delta_{y}^{2}\right) H_{y i+\frac{1}{2}, j}^{l} \\
& +a_{-} \delta_{y x} H_{x i+\frac{1}{2}, j}^{l}+b_{+} \bar{\delta}_{x y} E_{y i+\frac{1}{2}, j}^{l} \\
& -\frac{1}{2} \delta_{y}^{2} \bar{\delta}_{x y} E_{y i+\frac{1}{2}, j}^{l} \\
& \left(b_{+}^{2}-a_{-}^{2} \varepsilon_{r i, j+\frac{1}{2}}-\frac{b_{+}}{2} \bar{\delta}_{y}^{2}-\frac{b_{+}}{2} \delta_{x}^{2}+\frac{1}{4} \delta_{x}^{2} \bar{\delta}_{y}^{2}\right) E_{y i, j+\frac{1}{2}}^{l+1} \\
& =\left(b_{+} b_{-}-a_{+} a_{-} \varepsilon_{r i, j+\frac{1}{2}}\right. \\
& \left.+\frac{b_{+}}{2} \bar{\delta}_{y}^{2}-\frac{b_{-}}{2} \delta_{x}^{2}-\frac{1}{4} \delta_{x}^{2} \bar{\delta}_{y}^{2}\right) E_{y i, j+\frac{1}{2}}^{l} \\
& +\left(a_{+} b_{+}-a_{-} b_{-}-\frac{a_{+}+a_{-}}{2} \delta_{x}^{2}\right) H_{x i, j+\frac{1}{2}}^{l} \\
& -a_{-} \delta_{x y} H_{y i, j+\frac{1}{2}}^{l+1}+b_{+} \bar{\delta}_{y x} E_{x i, j+\frac{1}{2}}^{l+1} \\
& -\frac{1}{2} \delta_{x}^{2} \bar{\delta}_{y x} E_{x i, j+\frac{1}{2}}^{l+1} \\
& H_{x i, j+\frac{1}{2}}^{l+1} \\
& =\frac{1}{a_{-}}\left\{a_{+} H_{x i, j+\frac{1}{2}}^{l}-b_{+} E_{y i, j+\frac{1}{2}}^{l+1}+b_{-} E_{y i, j+\frac{1}{2}}^{l}\right. \\
& \left.+\frac{1}{2} \bar{\delta}_{y}^{2} E_{y i, j+\frac{1}{2}}^{l+1}+\frac{1}{2} \bar{\delta}_{y}^{2} E_{y i, j+\frac{1}{2}}^{l}+\bar{\delta}_{y x} E_{x i, j+\frac{1}{2}}^{l+1}\right\} \\
& H_{y i+\frac{1}{2}, j}^{l+1} \\
& =\frac{1}{a_{-}}\left\{a_{+} H_{y i+\frac{1}{2}, j}^{l}+b_{+} E_{x i+\frac{1}{2}, j}^{l+1}-b_{-} E_{x i+\frac{1}{2}, j}^{l}\right. \\
& \left.-\frac{1}{2} \bar{\delta}_{x}^{2} E_{x i+\frac{1}{2}, j}^{l+1}-\frac{1}{2} \bar{\delta}_{x}^{2} E_{x i+\frac{1}{2}, j}^{l}-\bar{\delta}_{x y} E_{y i+\frac{1}{2}, j}^{l}\right\}
\end{aligned}
$$

where

$$
\begin{aligned}
a_{ \pm} & =j k_{0}\left(1 / \Delta z \pm j \beta_{\text {ref }} / 2\right) \\
b_{ \pm} & =2 j \beta_{\text {ref }} / \Delta z \pm \beta_{\text {ref }}^{2} / 2 .
\end{aligned}
$$

For the unsplit-type, the solution can be updated by a single step unlike the split-type. It should be noted, however, that the bandwidth of the matrix is large. The matrix is calculated using the Bi-CGSTAB [17], resulting in a computational penalty, as will be seen in Fig. 5.

Fig. 4 denotes the error of $B$ against $\Delta$ for the quasi-TE and quasi-TM modes. The evaluation of the error is made by the difference between the calculated value $B_{\Delta}$ and the extrapolated 

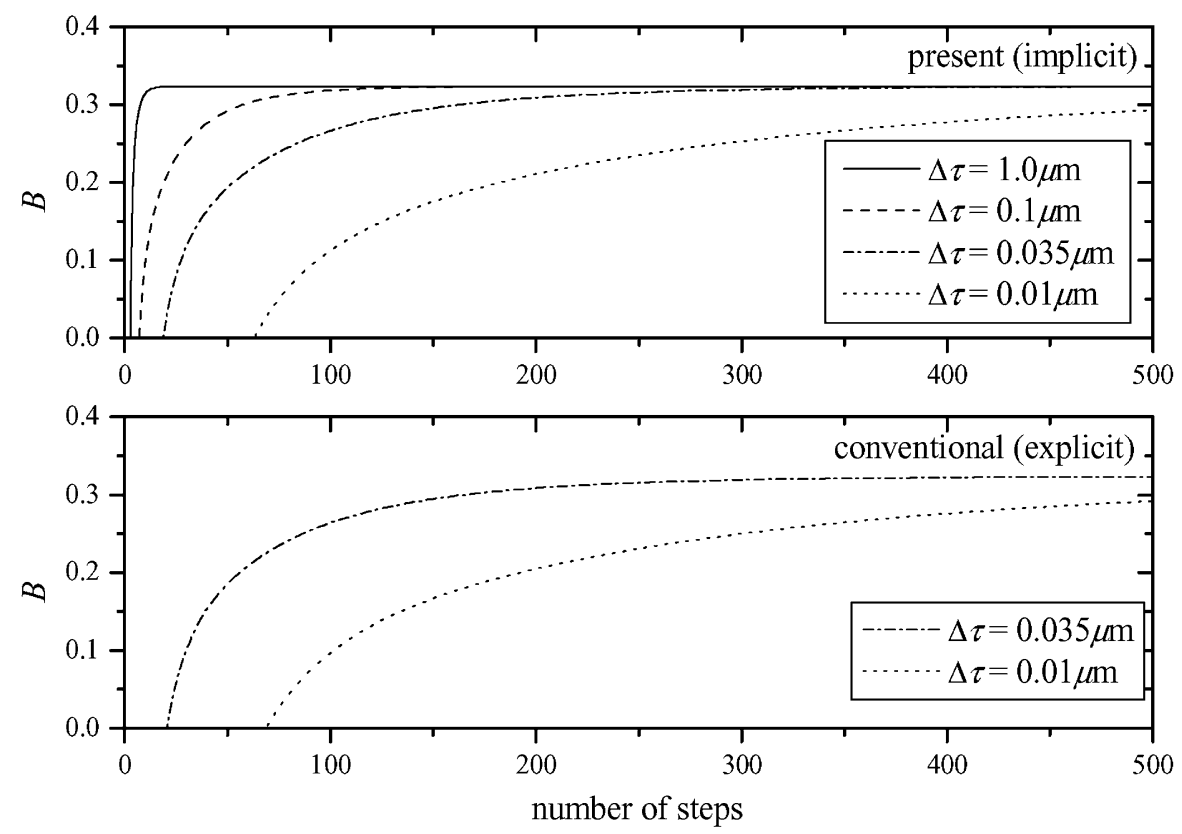

Fig. 3. Normalized propagation constant $B$ versus the number of steps.

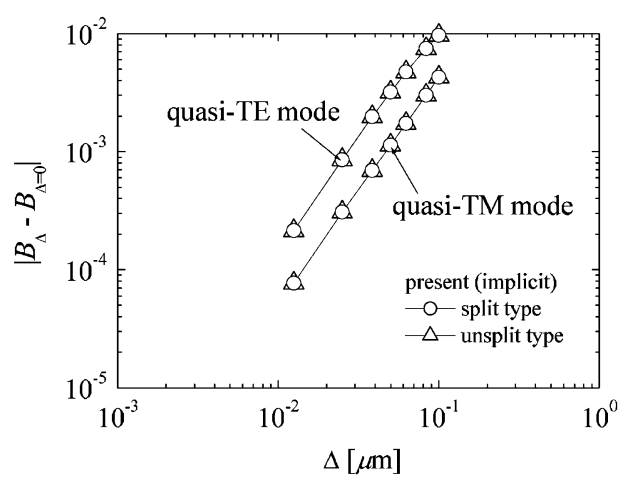

Fig. 4. Error of the normalized propagation constant versus the transverse sampling width.

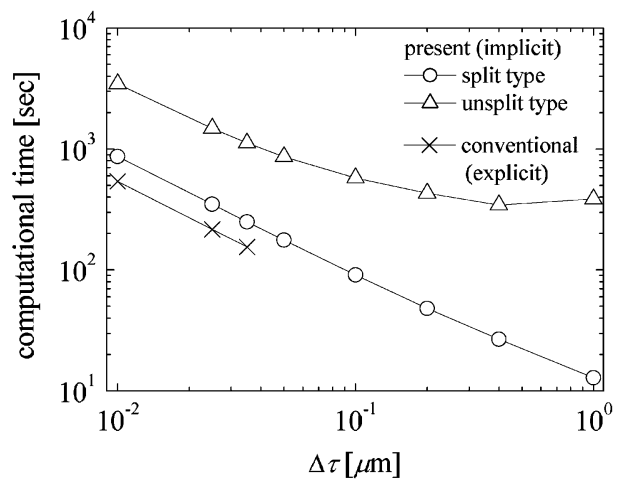

Fig. 5. Computational time versus the sampling width in the propagation direction.

value $B_{\Delta=0}$ considered as a true value. Good agreement is found between the results of the two (split- and unsplit-type) methods. Besides, it is clear that both methods ensure second-order accuracy. From the above results, it is concluded that the splitting procedure does not affect the accuracy of this analysis.

Next, we compare the computational time in order to reveal the effectiveness of splitting the propagation axis. Fig. 5

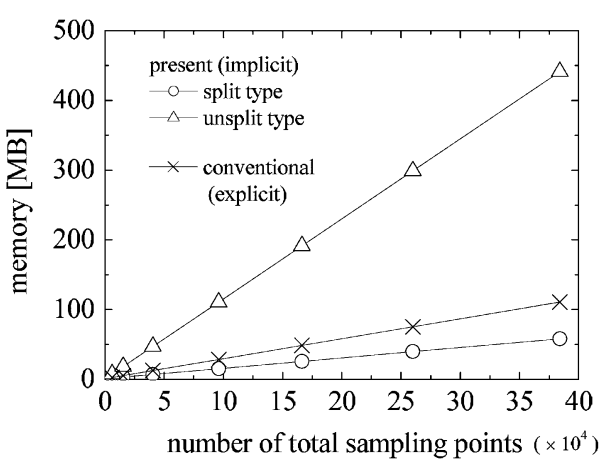

Fig. 6. Memory versus the number of total sampling points.

presents the computational time necessary to the convergence of $B$ versus $\Delta \tau$, in which $\Delta$ is fixed to be $0.05 \mu \mathrm{m}$. The calculation is carried out using the computer with a Pentium $42.26 \mathrm{GHz}$ processor. The computational time of the Bi-CGSTAB depends on a tolerance factor for convergence. To yield stable results, the tolerance factor is set to be $10^{-7}$. As expected, the computational time of the split-type is smaller than that of the unsplit type. When compared with the result of the conventional EY-BPM, the split-type is somewhat time consuming for the use of the same $\Delta \tau$. However, we can choose larger $\Delta \tau$ since the stability condition is removed in the split type. As a result, the computational time of the split type is reduced to $1 / 12$ in comparison with that of the conventional EY-BPM. Here, the computational time is defined as the time when the difference among $B$ s obtained from the four electromagnetic field components becomes less than or equal to $10^{-4}$. In this case, $B \mathrm{~s}$ calculated from the major components converge to a six-digit precision.

We finally investigate the memory requirement. Fig. 6 shows the required memory as a function of the number of total sampling points. It is found that the split type requires less memory. This is because the $E_{z}$ and $H_{z}$ components are transformed into the $E_{x}, E_{y}, H_{x}$, and $H_{y}$ components. 

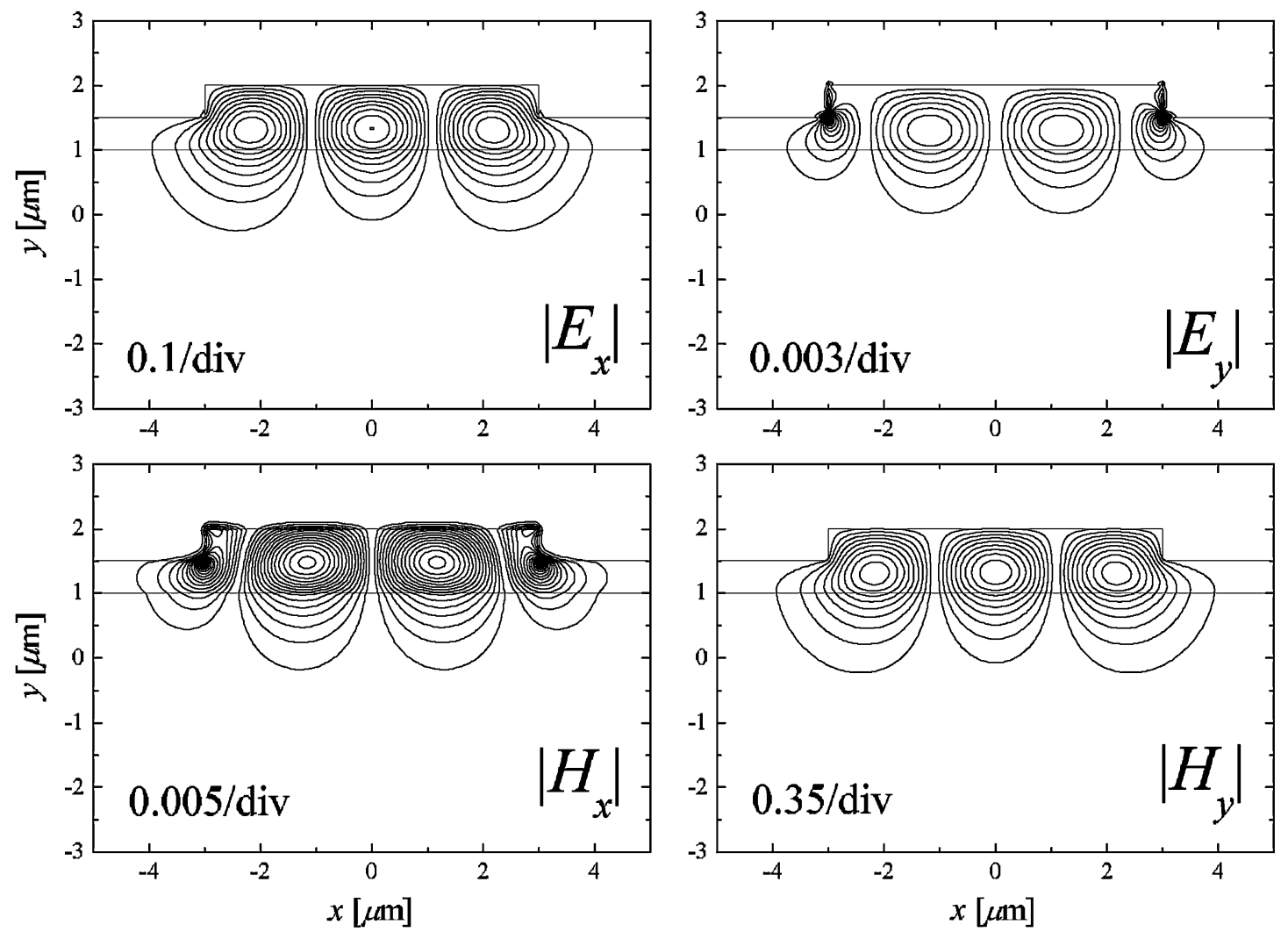

Fig. 7. Field profiles of the second-order quasi-TE mode.

\section{Higher Order Modes}

The fundamental modes of the rib waveguide have been treated in the above discussion. In this section, we extend the present method to the analysis of higher order modes. The higher order modes can be determined using the Gram-Schmidt orthogonalization technique, as follows:

$$
\phi_{\text {final }}(x, y, \tau) \equiv \phi(x, y, \tau)-\sum_{i=1}^{N} c^{i} \phi^{i}(x, y)
$$

where

$$
c^{i}=\frac{\int\left(\mathbf{E} \times \mathbf{H}^{i *}\right) \cdot \mathbf{n}_{z} d x d y}{\int\left(\mathbf{E}^{i} \times \mathbf{H}^{i *}\right) \cdot \mathbf{n}_{z} d x d y} .
$$

$\phi_{\text {final }}(x, y, \tau)$ is the field profile of the higher order eigenmode to be extracted, $\phi^{i}(x, y)$ is the field distribution of the $i$ th lowest order eigenmode, $\mathbf{n}_{z}$ is the unit vector in the $z$ direction, and * denotes the complex conjugate. The $N+1$ th mode is generated by subtracting the lower order eigenmodes $\phi^{i}(i=1, \ldots, N)$. Note that a similar expression is also used in [18] and [19].

We again analyze the rib waveguide shown in Fig. 1. The rib width $w$ is changed from $w=3.0 \mu \mathrm{m}$ to $w=6.0 \mu \mathrm{m}$ so that the second-order mode may be supported. The transverse sampling width is $\Delta=0.05 \mu \mathrm{m}$. The other parameters are identical with those shown in Section III.

Fig. 7 illustrates the field profiles of the second-order quasi-TE mode. Each electromagnetic field is normalized to the main component $E_{x}$. From the figure, we can see that the second-order mode profiles are successfully calculated.

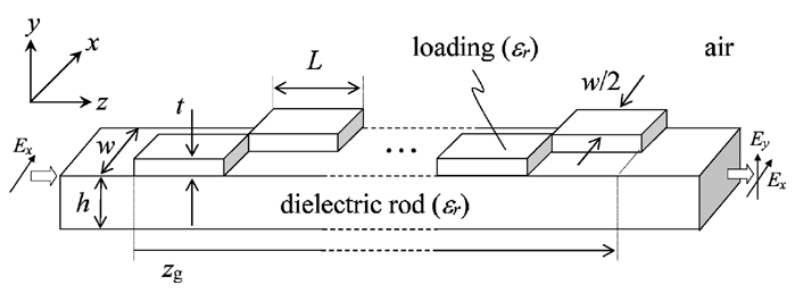

Fig. 8. Geometry of a polarization converter.

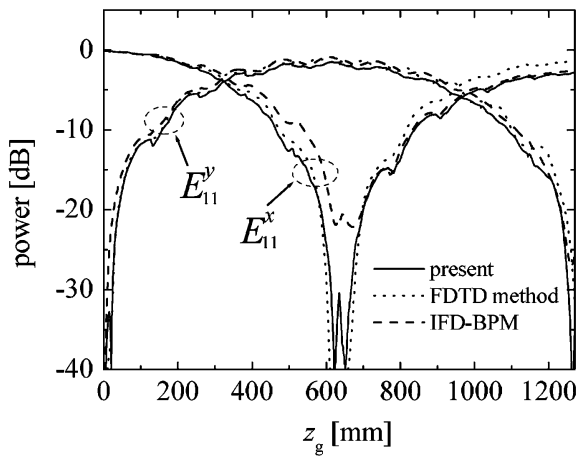

Fig. 9. Guided-mode power as a function of propagation distance.

\section{Propagating Beam Analysis}

The present method has the advantage that it can be applied to not only the eigenmode analysis but also the propagating beam analysis. In this section, the periodically loaded waveguide illustrated in Fig. 8, which is operated as a polarization converter, is analyzed as an example. Note that the device can 

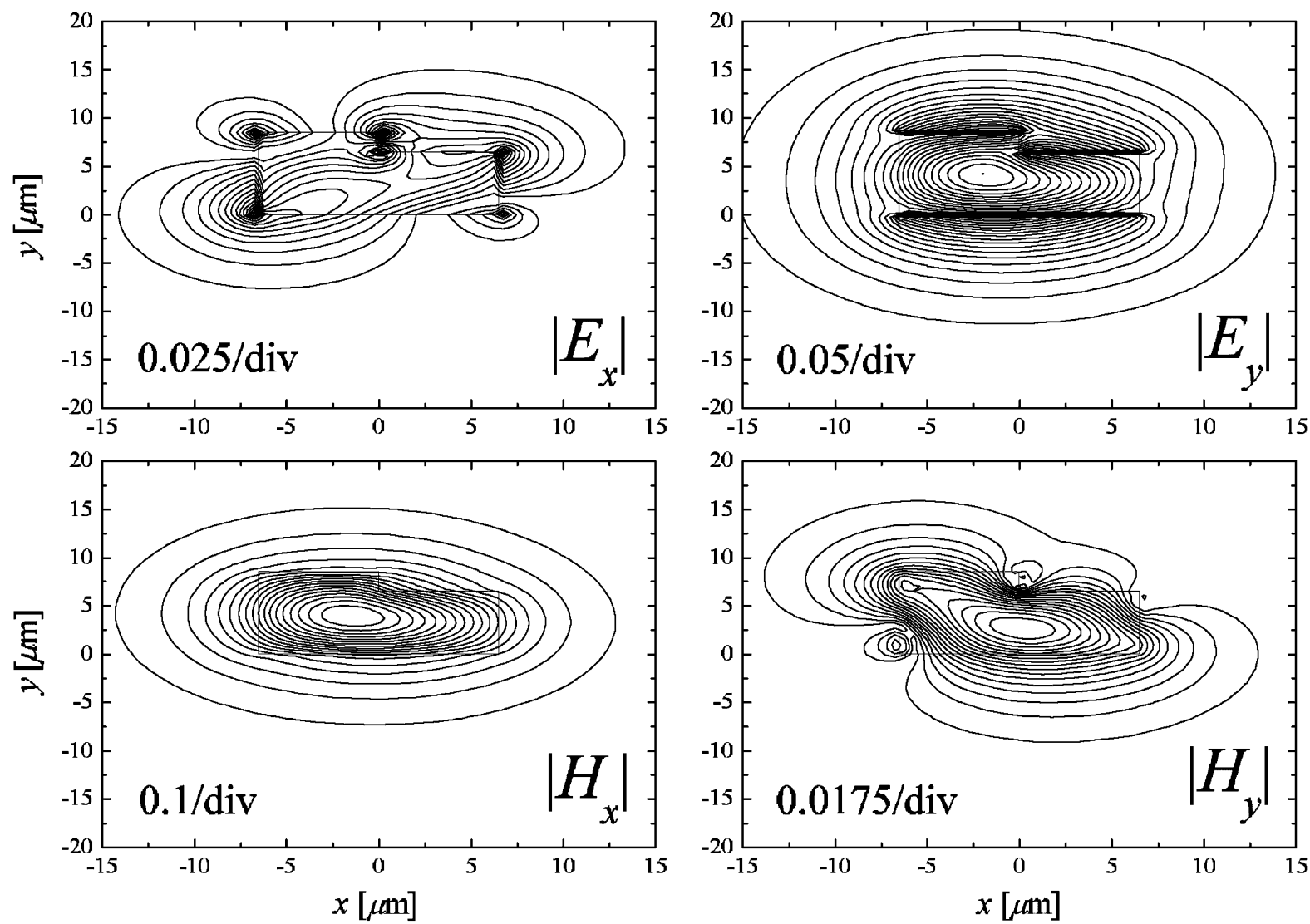

Fig. 10. Field profiles of the fundamental TM-like mode.

be geometrically expanded on the basis of a frequency transformation from optical to microwave frequencies. Therefore, the operating wavelength is taken to be $\lambda=20 \mathrm{~mm}$, which is determined by the previous experimental investigation [20]. The relative permittivity of the dielectric rod and loadings is $\varepsilon_{r}=2.8$. The dimensions of the $x-y$ plane of the rod are fixed to be $w \times h=13.0 \mathrm{~mm} \times 6.5 \mathrm{~mm}$. The width and thickness of the loading are $w / 2=6.5$ and $t=2.0 \mathrm{~mm}$, respectively. The loading length is $L=127.0 \mathrm{~mm}$.

The sampling widths are chosen to be $\Delta=0.5 \mathrm{~mm}$ and $\Delta z=1.0 \mathrm{~mm}$. The number of transverse sampling points is $N_{x} \times N_{y}=150 \times 150$. The PML is employed to absorb outgoing waves at the edge of the computational domain. The rod is excited with the $E_{11}^{x}$ (TE) mode whose field profiles are obtained with the imaginary-distance procedure. It is expected that the $E_{11}^{x}$ mode is progressively converted into the $E_{11}^{y}(\mathrm{TM})$ mode as the wave propagates along the polarization converter.

Fig. 9 represents the guided-mode power as a function of propagation distance $z_{g}$. The guided-mode power is normalized to the excited $E_{11}^{x}$ mode power. Almost complete polarization conversion is obtained at $z_{g}=635 \mathrm{~mm}$. For comparison, the data calculated by the finite-difference time-domain (FDTD) method and the full-vectorial BPM with the improved finite-difference formula (IFD-BPM) [11] are presented using dotted and dashed lines. It is seen that the result of the present method agrees with those of the FDTD method and IFD-BPM. To be exact, the guided-mode power obtained from the present method and IFD-BPM is slightly smaller (approximately $1.4 \mathrm{~dB}$ ) than that of the FDTD method. We should recall, however, that the

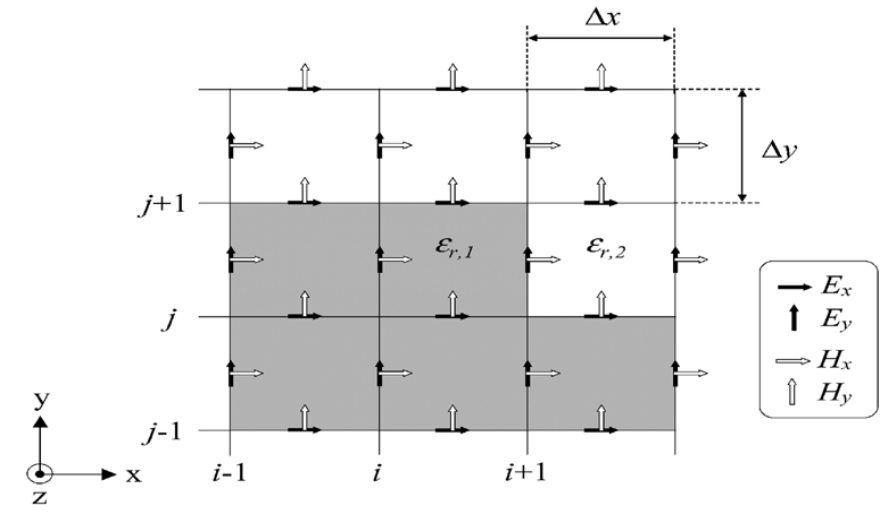

Fig. 11. Yee's mesh.

BPMs are much more efficient in terms of computational time and memory than the FDTD method [20].

To validate a conversion length of $635 \mathrm{~mm}$, we calculate the eigenmodes of the polarization converter. The Gram-Schmidt orthogonalization technique is again used to obtain the TM-like mode, since its propagation constant is smaller than that of the TE-like mode. More specifically, we first calculate the TE-like mode field, which has the largest propagation constant, and then the TM-like mode field is calculated, while subtracting the previously determined TE-like mode field. As an example, the fields of the TM-like mode are displayed in Fig. 10. From the obtained propagation constants $\left(\beta_{\mathrm{TE}}\right.$ and $\left.\beta_{\mathrm{TM}}\right)$, we can evaluate the conversion length $L_{\mathrm{con}}$ from $L_{\mathrm{con}}=\pi /\left(\beta_{\mathrm{TE}}-\beta_{\mathrm{TM}}\right)$. 


$$
\begin{aligned}
\bar{\delta}_{x}^{2} E_{x i+\frac{1}{2}, j} & =\frac{c_{1} E_{x i-\frac{1}{2}, j}-c_{2} E_{x i+\frac{1}{2}, j}+c_{3} E_{x i+\frac{3}{2}, j}}{\Delta x^{2}} \\
\bar{\delta}_{y}^{2} E_{y i, j+\frac{1}{2}} & =\frac{c_{4} E_{y i, j-\frac{1}{2}}-c_{5} E_{y i, j+\frac{1}{2}}+c_{6} E_{y i, j+\frac{3}{2}}}{\Delta y^{2}} \\
\delta_{x}^{2} H_{x i, j+\frac{1}{2}} & =\frac{H_{x i-1, j+\frac{1}{2}}-2 H_{x i, j+\frac{1}{2}}+H_{x i+1, j+\frac{1}{2}}}{\Delta x^{2}} \\
\delta_{y}^{2} H_{y i+\frac{1}{2}, j} & =\frac{H_{y i+\frac{1}{2}, j-1}-2 H_{y i+\frac{1}{2}, j}+H_{y i+\frac{1}{2}, j+1}}{\Delta y^{2}} \\
\bar{\delta}_{y x} E_{x i, j+\frac{1}{2}} & =\frac{d_{1} E_{x i-\frac{1}{2}, j}-d_{2} E_{x i+\frac{1}{2}, j}-d_{3} E_{x i-\frac{1}{2}, j+1}+d_{4} E_{x i+\frac{1}{2}, j+1}}{\Delta x \Delta y} \\
\bar{\delta}_{x y} E_{y i+\frac{1}{2}, j} & =\frac{d_{5} E_{y i, j-\frac{1}{2}}-d_{6} E_{y i, j+\frac{1}{2}}-d_{7} E_{y i+1, j-\frac{1}{2}}+d_{8} E_{y i+1, j+\frac{1}{2}}}{\Delta x \Delta y} \\
\delta_{y x} H_{x i+\frac{1}{2}, j} & =\frac{H_{x i, j-\frac{1}{2}}-H_{x i, j+\frac{1}{2}}-H_{x i+1, j-\frac{1}{2}}+H_{x i+1, j+\frac{1}{2}}}{\Delta x \Delta y} \\
\delta_{x y} H_{y i, j+\frac{1}{2}} & =\frac{H_{y i-\frac{1}{2}, j}-H_{y i-\frac{1}{2}, j+1}-H_{y i+\frac{1}{2}, j}+H_{y i+\frac{1}{2}, j+1}}{\Delta x \Delta y}
\end{aligned}
$$

It is confirmed that the evaluated conversion length $(635 \mathrm{~mm})$ is identical with that observed in the propagating beam analysis in Fig. 9.

Finally, we check the accuracy of the present method using the Y junction splitter considered in [21], [22]. Since the splitter is a longitudinally variant waveguide, we adopt an index averaging technique. Calculation shows that the difference between the present and FDTD methods in the normalized power of the lowest order local normal mode is within \pm 0.02 for both $E^{x}$ and $E^{y}$ modes. We should, however, note that the present formulation is based on the Fresnel-type equations, so that the accuracy will be lost, as the longitudinal variation of the waveguide becomes large and the propagating field varies rapidly. In the present $\mathrm{Y}$-junction splitter, an average half-branching angle is around $3^{\circ}$. Further calculation shows that the error becomes large as the branching angle is increased. Roughly speaking, the branching angle must be less than $5^{\circ}$ to maintain reasonable accuracy.

\section{CONCLUSION}

An implicit Yee-mesh-based finite-difference full-vectorial beam-propagation method (BPM) has been proposed for the eigenmode and propagating beam analyzes of optical waveguides. We first discuss the formulation in detail and then perform the eigenmode analysis of a rib waveguide with the imaginary-distance procedure. After confirming the validity of the present method, it is revealed that the computational time of the present method is reduced to $1 / 12$ in comparison with that of the conventional explicit Yee-mesh-based imaginary-distance BPM, with the required memory also being reduced. Furthermore, the higher order mode is generated using the full-vectorial Gram-Schmidt orthogonalization technique. Finally, it is demonstrated through the analysis of a polarization converter that the present method can be used for not only the eigenmode analysis but also the propagating beam analysis.

\section{APPENDIX}

Referring to Fig. 11, we express the difference operators as (40)-(47), shown at the top of the page, where

$$
\begin{aligned}
& c_{1}=\frac{\varepsilon_{r i-\frac{1}{2}, j}}{\varepsilon_{r i, j}}, \quad c_{2}=\frac{\varepsilon_{r i+\frac{1}{2}, j}}{\varepsilon_{r i, j}}+\frac{\varepsilon_{r i+\frac{1}{2}, j}}{\varepsilon_{r i+1, j}}, \quad c_{3}=\frac{\varepsilon_{r i+\frac{3}{2}, j}}{\varepsilon_{r i+1, j}} \\
& c_{4}=\frac{\varepsilon_{r i, j-\frac{1}{2}}}{\varepsilon_{r i, j}}, \quad c_{5}=\frac{\varepsilon_{r i, j+\frac{1}{2}}}{\varepsilon_{r i, j}}+\frac{\varepsilon_{r i, j+\frac{1}{2}}}{\varepsilon_{r i, j+1}}, \quad c_{6}=\frac{\varepsilon_{r i, j+\frac{3}{2}}}{\varepsilon_{r i, j+1}} \\
& d_{1}=\frac{\varepsilon_{r i-\frac{1}{2}, j}}{\varepsilon_{r i, j}}, \quad d_{2}=\frac{\varepsilon_{r i+\frac{1}{2}, j}}{\varepsilon_{r i, j}}, \quad d_{3}=\frac{\varepsilon_{r i-\frac{1}{2}, j+1}}{\varepsilon_{r i, j+1}}, \\
& d_{4}=\frac{\varepsilon_{r i+\frac{1}{2}, j+1}}{\varepsilon_{r i, j+1}}, \quad d_{5}=\frac{\varepsilon_{r i, j-\frac{1}{2}}}{\varepsilon_{r i, j}}, \quad d_{6}=\frac{\varepsilon_{r i, j+\frac{1}{2}}}{\varepsilon_{r i, j}}, \\
& d_{7}=\frac{\varepsilon_{r i+1, j-\frac{1}{2}}}{\varepsilon_{r i+1, j}}, \quad d_{8}=\frac{\varepsilon_{r i+1, j+\frac{1}{2}}}{\varepsilon_{r i+1, j}} .
\end{aligned}
$$

\section{ACKNOWLEDGMENT}

The authors would like to thank H. Nakayama for his basic investigations of the present work.

\section{REFERENCES}

[1] M. Koshiba, Optical Waveguide Analysis. New York: McGraw-Hill, 1990.

[2] D. Yevick and W. Bardyszewski, "Correspondence of variation finitedifference (relaxation) and imaginary-distance propagation methods for modal analysis," Opt. Lett., vol. 17, no. 5, pp. 329-330, 1992.

[3] C. Vassallo, "1993-1995 Optical mode solvers," Opt. Quantum Electron., vol. 29, pp. 95-114, 1997.

[4] S. M. Lee, "Finite-difference vectorial-beam-propagation method using Yee's discretization scheme for modal fields," J. Opt. Soc. Amer. A, Opt. Image Sci., vol. 13, no. 7, pp. 1369-1377, 1996.

[5] T. Ando, H. Nakayama, S. Numata, J. Yamauchi, and H. Nakano, "Eigenmode analysis of optical waveguides by a Yee-mesh-based imaginary-distance propagation method for an arbitrary dielectric interface," J. Lightw. Technol., vol. 20, no. 8, pp. 1627-1634, Aug. 2002.

[6] T. Ando, J. Yamauchi, and H. Nakano, "Numerical analysis of a dielectric rod antenna-Demonstration of the discontinuity-radiation concept," IEEE Trans. Antennas Propagat., vol. 51, no. 8, pp. 2007-2013, Aug. 2003. 
[7] J. Yamauchi, T. Mugita, T. Ando, H. Nakayama, and H. Nakano, "Yeemesh-based real axis beam-propagation method," Proc. 3rd Int. Workshop on Scientific Computing Applications, pp. 47-48, 2003.

[8] S. T. Chu, W. P. Huang, and S. K. Chaudhuri, "Simulation and analysis of waveguide based optical integrated circuits," Comp. Phys. Commun., vol. 68, pp. 451-484, 1991.

[9] M. S. Stern, "Semivectorial polarized finite difference method for optical waveguides with arbitrary index profiles," Proc. Inst. Elect. Eng. J, vol. 135, no. 1, pp. 56-63, 1988.

[10] W. P. Huang and C. L. Xu, "Simulation of three-dimensional optical waveguides by a full-vector beam propagation method," IEEE J. Quantum Electron., vol. 29, no. 10, pp. 2639-2649, Oct. 1993.

[11] J. Yamauchi, Propagating Beam Analysis of Optical Waveguides. Herfordshire, U.K.: Research Studies Press, Ltd., 2003.

[12] G. R. Hadley, "Transparent boundary condition for beam propagation," Opt. Lett., vol. 16, no. 9, pp. 624-626, 1991.

[13] J. P. Berenger, "A perfectly matched layer for the absorption of electromagnetic waves," J. Comput. Phys., vol. 114, pp. 185-200, 1994.

[14] W. H. Press, S. A. Teukolsky, W. T. Vetterling, and B. P. Flannery, Numerical Recipes. Cambridge, U.K.: Cambridge Univ. Press, 1992.

[15] C. L. Xu, W. P. Huang, and S. K. Chaudhuri, "Efficient and accurate vector mode calculations by beam propagation method," J. Lightw. Technol., vol. 11, no. 7, pp. 1209-1215, Jul. 1993.

[16] S. Jüngling and J. C. Chen, "A study and optimization of eigenmode calculations using the imaginary-distance beam-propagation method," IEEE J. Quantum Electron., vol. 30, no. 9, pp. 2098-2105, Sep. 1994.

[17] H. A. Van Der Vorst, "Bi-CGSTAB: A fast and smoothly converging variant of Bi-CG for the solution of nonsymmetric linear system," SIAM J. Sci. Stat. Comput., vol. 13, pp. 631-644, 1992.

[18] S. S. A. Obayya, B. M. A. Rahman, K. T. V. Grattan, and H. A. El-Mikati, "Full vectorial finite-element-based imaginary distance beam propagation solution of complex modes in optical waveguides," J. Lightw. Technol., vol. 20, no. 6, pp. 1054-1060, Jun. 2002.

[19] K. Saitoh and M. Koshiba, "Full-vectorial imaginary-distance beam propagation method based on a finite element scheme: Application to photonic crystal fibers," IEEE J. Quantum Electron., vol. 38, no. 7, pp. 927-933, Jul. 2002

[20] T. Ando, T. Murata, H. Nakayama, J. Yamauchi, and H. Nakano, "Analysis and measurement of polarization conversion in a periodically loaded dielectric waveguide," IEEE Photon. Technol. Lett., vol. 14, no. 9, pp. 1288-1290, Sep. 2002.

[21] Y. Tsuji, M. Koshiba, and T. Shiraishi, "Finite-element beam propagation method for three-dimensional optical waveguide structures," $J$. Lightw. Technol., vol. 15, no. 9, pp. 1728-1734, Sep. 1997.

[22] J. P. da Silva, H. E. Hernández-Figueroa, and A. M. F. Frasson, "Improved vectorial finite-element BPM analysis for transverse anisotropic media," J. Lightw. Technol., vol. 21, no. 2, pp. 567-576, Feb. 2003.

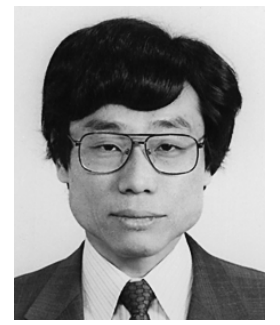

Junji Yamauchi (M'85) was born in Nagoya, Japan, on August 23, 1953. He received the B.E., M.E., and Dr.Eng. degrees from Hosei University, Tokyo, Japan, in 1976, 1978, and 1982, respectively.

From 1984 to 1988 , he served as a Lecturer in the Electrical Engineering Department of Tokyo Metropolitan Technical College. Since 1988, he has been a Member of the Faculty of Hosei University, where he is now a Professor of electronic informatics. His research interests include optical waveguides and circularly polarized antennas. $\mathrm{He}$ is the author of the book Propagating Beam Analysis of Optical Waveguides (Hertfordshire, U.K.: Research Studies Press, 2003).

Dr. Yamauchi is a Member of the Optical Society of America (OSA) and the Institute of Electronics, Information and Communication Engineers (IEICE) of Japan.

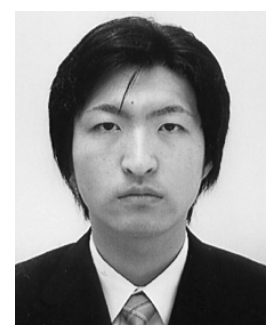

Takanori Mugita was born in Saitama, Japan, on May 28, 1979. He received the B.E. and M.E. degrees from Hosei University, Tokyo, Japan, in 2002 and 2004, respectively.

His research interests include numerical analysis of optical waveguides. He joined Fujitsu, Limited, Tokyo, Japan, in 2004

Mr. Mugita is a Member of the Institute of Electronics, Information and Communication Engineers (IEICE) of Japan.

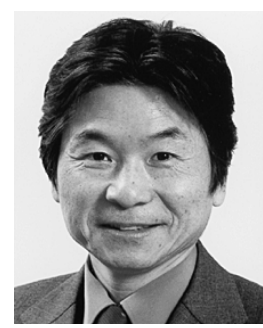

Hisamatsu Nakano (M'75-SM'87-F'92) was born in Ibaraki, Japan, on April 13, 1945. He received the B.E., M.E., and Dr.Eng. degrees in electrical engineering from Hosei University, Tokyo, Japan, in 1968, 1970, and 1974, respectively.

Since 1973, he has been a Member of the Faculty of Hosei University, where he is now a Professor of Electronic Informatics. His research topics include numerical methods for low- and high-frequency antennas and optical waveguides. He has published more than 190 refereed journal papers and more than 150 international symposium papers. He is the author of the book Helical and Spiral Antennas (New York: Research Studies Press, Wiley, 1987) and the coauthor of Analysis Methods of Electromagnetic Wave Problems, Volume Two (Norwood, MA: Artech House, 1996). In addition, he is the author of Helical and Spiral Antennas, Encyclopedia of Telecommunications (New York: Wiley, 2002).

Dr. Nakano received the Institution of Electrical Engineers (IEE) International Conference on Antennas and Propagation Best Paper Award and the IEEE TRAnSACtions on ANTENNAS and Propagation Best Application Paper Award (H. A. Wheeler Award) in 1989 and 1994, respectively. In 2001, he received the Award of Distinguished Technical Communication (from the Society for Technical Communication) and the Science and Technology Progress Award (Hangzhou, China). 